\title{
INFINITESIMAL PSEUDO-METRICS AND THE SCHWARZ LEMMA
}

\author{
M. KLIMEK
}

(Communicated by Irwin Kra)

\begin{abstract}
In this paper we investigate the relationship between infinitesimal pseudo-metrics introduced by N. Sibony and K. Azukawa. Also we prove a version of the Schwarz lemma for plurisubharmonic functions.
\end{abstract}

\section{INTRODUCTION}

Let $M$ be a complex manifold. Throughout the paper we shall be assuming that the dimension of $M$ is $n$. Sibony in [8] and Azukawa in [1] introduced infinitesimal pseudo-metrics on the tangent bundle $T M$ using families of bounded plurisubharmonic functions on $M$. We shall denote the pseudometrics by $S_{M}$ and $A_{M}$ respectively (the definitions of the pseudo-metrics are given in the next section). Both $S_{M}$ and $A_{M}$ contract holomorphic mappings and hence $C_{M} \leq S_{M} \leq K_{M}$ and $C_{M} \leq A_{M} \leq K_{M}$ where $C_{M}$ and $K_{M}$ denote the Caratheodory and Kobayashi infinitesimal pseudo-metrics respectively. The purpose of this paper is to study the relationship between $S_{M}$ and $A_{M}$.

In order to be able to state the main results we need to recall the definition of an extremal plurisubharmonic function, originally introduced in [6] and then studied in $[2,4]$.

Let $\mathscr{P}(M, p)$ denote the family of all negative plurisubharmonic functions $u$ on $M$ such that for any holomorphic chart $\varphi: U \rightarrow \varphi(U) \subset \mathbf{C}^{n}$ where $p \in U \subset M$ and $\varphi(p)=0$, the function $u \circ \varphi^{-1}-\log \|\cdot\|$ is bounded from above in a neighbourhood of 0 . (By $\|\cdot\|$ we denote the Euclidean norm in $\mathbf{C}^{n}$.) Define

$$
u_{M}(z, p)=\sup \{u(z): u \in \mathscr{P}(M, p)\} .
$$

It can be proved that $u_{M}(\cdot, p) \in \mathscr{P}(M, p)$. Moreover, if $M$ is an open subset of $\mathbf{C}$ such that $u_{M}(z, p) \rightarrow 0$ as $z \rightarrow \partial M$, then $-u_{M}(\cdot, p)$ coincides with the Green function for $M$ with pole at $p$. For further properties of the extremal function $u_{M}$ and its applications in complex analysis see [6, 4, 2].

Received by the editors August 4, 1987 and, in revised form, February 12, 1988.

1980 Mathematics Subject Classification (1985 Revision). Primary 32H15, 32F05. 
Let $\mathscr{C}^{2}(\{p\})$ denote the family of all functions which are of class $\mathscr{C}^{2}$ in some neighbourhood of $p$.

We show the following.

Theorem 1. If $M$ is a complex manifold then $S_{M} \leq A_{M}$. If $M$ is a Stein manifold then $A_{M}$ is upper semicontinuous and hence $S_{M}^{*} \leq A_{M}$ (where the asterisk denotes the upper-semicontinuous regularization). If $\exp \left(2 u_{M}(\cdot, p)\right) \in$ $\mathscr{C}^{2}(\{p\})$ for some $p \in M$ then

$$
S_{M}(\xi)=A_{M}(\xi)=\left\langle\mathscr{L}\left(\exp \left(2 u_{M}(\cdot, p)\right)\right)(p) \xi, \xi\right\rangle^{1 / 2}, \quad \xi \in T_{p} M,
$$

where $T_{p} M$ is the tangent space to $M$ at $p$ and $\langle\mathscr{L} \cdot, \cdot\rangle$ denotes the Levi form.

If $P_{M}$ is an infinitesimal pseudometric on $M$ and $p \in M$, the set $I_{p}\left(P_{M}\right)=$ $\left\{v \in T_{p} M: P_{M}(v)<1\right\}$ is called the indicatrix of $P_{M}$ at $p$. It can be shown that the indicatrices of $S_{M}$ are always convex (see [8]) and those of $A_{M}$ arein general-only starlike circular. Therefore, it is easy to furnish examples of manifolds $M$ for which the two pseudo-metrics differ. For instance we can take a plurisubharmonic function $h: \mathbf{C}^{n} \rightarrow \mathbf{R}_{+}$such that $h(\lambda z)=|\lambda| h(z)$ for $\lambda \in \mathbf{C}, z \in \mathbf{C}^{n}$ and $M=\left\{z \in \mathbf{C}^{n}: h(z)<1\right\}$ is not convex (e.g. $h\left(z_{1}, z_{2}\right)=$ $\max \left\{\left|z_{1}\right|,\left|z_{2}\right|, 2 \sqrt{\left|z_{1} z_{2}\right|}\right\}$ for $\left.\left(z_{1}, z_{2}\right) \in \mathbf{C}^{2}\right)$. Then $A_{M}(0, \xi)=h(\xi)$ (see [1]) and hence $A_{M} \neq S_{M}$ on $T_{0} M \equiv \mathrm{C}^{n}$. An example of a nonconvex $M$ for which $S_{M}=A_{M}$ is provided by the open annulus $M=\{z \in \mathbf{C}: r<|z|<R\}$ where $r>0, R>0$. The equality follows from Theorem 1 because $\exp \left(2 u_{M}(\cdot, p)\right) \in$ $\mathscr{C}^{2}(M)$ (see [6]).

We also prove a version of the Schwarz lemma for plurisubharmonic functions. To state the result, we have to introduce some notation. Let $p \in M$ and let $\mathscr{S}(M, p)$ denote the family of all logarithmically plurisubharmonic functions $u: M \rightarrow[0,1]$ such that $u(p)=0$ and $u \in \mathscr{C}^{2}(\{p\})$. By $\mathscr{F}(M, p)$ we will denote the set of all plurisubharmonic functions $u$ on $M$ with the property that for each $z \in M \backslash\{p\}$ there exists a connected one-dimensional complex submanifold $N$ of $M$ such that $z, p \in N$ and the restriction of $u$ to $N \backslash\{p\}$ is harmonic.

Theorem 2. Let $\Omega$ be a relatively compact open connected subset of a Stein manifold $M$ and let $u_{\Omega}=u_{\Omega}(\cdot, p)$. If $v \in \mathscr{S}(\Omega, p)$ then

$$
v \leq \exp \left(2 u_{\Omega}\right) \quad \text { in } \Omega \backslash\{p\} \text {. }
$$

Moreover if $\exp \left(2 u_{\Omega}\right) \in \mathscr{C}^{2}(\{p\})$ then for all $\xi \in T_{p} M$

$$
\langle\mathscr{L} v(p) \xi, \xi\rangle \leq\left\langle\mathscr{L}\left(\exp \left(2 u_{\Omega}\right)\right)(p) \xi, \xi\right\rangle \text {. }
$$

If $u_{\Omega} \in \mathscr{F}(\Omega, p)$ and the equality holds in (4) for all $\xi \in T_{p} M$ then $v \equiv$ $\exp \left(2 u_{\Omega}\right)$. If $n=1$ and the equality holds in (3) for one $z \in \Omega$ then $v \equiv$ $\exp \left(2 u_{\Omega}\right)$.

When $\Omega$ is equal to the unit disc, $\exp \left(2 u_{\Omega}(z)\right)=|z|^{2}$ and hence the theorem reduces to the version of the Schwarz lemma proved by Sibony in [8]. 
It is easy to notice that the last conclusion of the theorem is not true for $n>1$. For instance, if $\Omega$ is the open unit ball in $C^{2}, p$ is the origin and $v\left(z_{1}, z_{2}\right)=\left|z_{1}\right|^{2}+a\left|z_{2}\right|^{2}$ (where $a \in[0,1)$ is fixed) then $v \not \equiv \exp \left(2 u_{\Omega}\right)=\|\cdot\|^{2}$.

Note also that if $n=1, \exp 2 u_{\Omega} \in \mathscr{C}^{2}(\{p\})$. This is so, because $-u_{\Omega}$ is the generalized Green function for $\Omega$ with pole at $p$.

Assume now that $\Omega \subset M$ is such that $u=u_{\Omega}(\cdot, p)$ is a $\mathscr{C}^{2}$-function on $\Omega \backslash\{p\}$ and $u(z) \rightarrow 0$ as $z$ approaches the boundary of $\Omega$. It is known that $u$ satisfies the homogeneous Monge-Ampère equation $\left(d d^{c} u\right)^{n}=0$ in $\Omega \backslash\{p\}$ (see [6]). If we also assume that $\left(d d^{c} u\right)^{n-1} \neq 0$ at each point of $\Omega \backslash\{p\}$, then-in view of [3]-there is a foliation of $\Omega \backslash\{p\}$ by one-dimensional complex manifolds with the property that the restriction of $u$ to each of them is harmonic (an alternative proof can be found in [5]). As observed in [4], it follows from the maximum principle that if $N$ is a leaf of the foliation, then $p \in \bar{N}$ and $\bar{N} \cap \Omega$ is a one-dimensional analytic subvariety of $\Omega$. It would be interesting to know under what condition $p$ is a regular point of $\bar{N} \cap \Omega$ for each leaf $N$ of the foliation. (If this was the case, $u$ would be a member of $\mathscr{F}(\Omega, p)$.) Under the assumption that $\Omega$ is a bounded strictly convex domain in $\mathbf{C}^{n}$, an affirmative answer follows from Lempert's study of the Kobayashi metric [7]. It has been conjectured by Demailly [4] that if $\Omega$ is strictly pseudoconvex then the above conditions are also met-i.e. $u$ is smooth on $\Omega \backslash\{p\}, d d^{c} u$ has constant rank $n-1$ in $\Omega \backslash\{p\}$ and the leaves of the associated foliation extend through $p$ to complex submanifolds of $\Omega$.

In the light of the above remarks, the assumption that $u_{\Omega} \in \mathscr{F}(\Omega, p)$ does not seem to be too restrictive.

\section{The families of FUnCtions $\mathscr{P}(M, p)$ AND $\mathscr{S}(M, p)$}

In this section we shall establish the relationship between the two families of plurisubharmonic functions defined in the introduction.

The following lemma follows directly from the proof of a version of the Schwarz lemma obtained by Sibony in [8]. For the sake of completeness we give a proof here.

Lemma 1. Let $D \subset \mathbf{C}$ be a neighbourhood of zero. If $u \in \mathscr{S}(D, 0)$ then

$$
u(z)=\frac{1}{4}(\Delta u)(0)|z|^{2}+o\left(|z|^{2}\right), \quad \text { as } z \rightarrow 0 .
$$

Proof. In view of Taylor's formula for $u$ at $0, v(z)=\left(u(z) /|z|^{2}\right)^{*}$ is subharmonic in $D$ and

$$
\lim _{t \rightarrow 0^{+}} \frac{u(t \alpha, t \beta)}{t^{2}}=\frac{1}{2} \frac{\partial^{2} u}{\partial x^{2}}(0) \alpha^{2}+\frac{\partial^{2} u}{\partial x \partial y}(0) \alpha \beta+\frac{1}{2} \frac{\partial^{2} u}{\partial y^{2}}(0) \beta^{2}
$$

for any $\alpha+i \beta$ from the unit circle. Moreover, as closed line segments in $\mathbf{C}$ are not thin, the limit on the left hand side of the above equality is $v(0)$. By 
taking $\alpha+i \beta$ equal to $1, i$ and $(1+i) / \sqrt{2}$ we conclude that

$$
\frac{\partial^{2} u}{\partial x^{2}}(0)=\frac{\partial^{2} u}{\partial y^{2}}(0) \quad \text { and } \quad \frac{\partial^{2} u}{\partial x \partial y}(0)=0
$$

Hence the Taylor expansion of $u$ at 0 looks exactly as stated in our lemma.

Let $M$ be a complex manifold and let $p \in M$. For $u \in \mathscr{C}^{2}(\{p\})$ one can define the Levi form $\langle\mathscr{L} u(p) \cdot, \cdot\rangle$ of $u$ at $p$ as follows. Let $\xi \in T_{p} M$ and let $\varphi: U \rightarrow \varphi(U) \subset \mathbf{C}^{n}$ be a chart on $M$ in a neighbourhood $U$ of $p$ such that $\varphi(p)=0$. If $\left(\zeta_{1}, \ldots, \zeta_{n}\right)=d_{p} \varphi(\xi)$ then we put

$$
\langle\mathscr{L} u(p) \xi, \xi\rangle=\sum_{i, j=1}^{n} \frac{\partial^{2}\left(u \circ \varphi^{-1}\right)(0)}{\partial z_{i} \partial \bar{z}_{j}} \zeta_{i} \bar{\zeta}_{j} .
$$

It is easy to show that this definition is independent of the choice of $\varphi$.

Lemma 2. If $u \in \mathscr{S}(M, p)$ then $\log \sqrt{u} \in \mathscr{P}(M, p)$. Moreover if $V \subset \mathbf{C}$ is a neighbourhood of the origin and $F: V \rightarrow M$ is a holomorphic mapping such that $F(0)=p$ and $F^{\prime}(0)=\xi$ we have

$$
\langle\mathscr{L} u(p) \xi, \xi\rangle=\lim _{\substack{\lambda \rightarrow 0 \\ \lambda \in C}} \frac{(u \circ F)(\lambda)}{|\lambda|^{2}} .
$$

Proof. Without loss of generality we may assume that $M$ is an open subset of $\mathbf{C}^{n}$ and $p=0$. Since $\langle\mathscr{L} u(p) \xi, \xi\rangle=\left.\frac{1}{4} \Delta(\lambda \rightarrow(u \circ F)(\lambda))\right|_{\lambda=0}($ where $\lambda \in \mathbf{C})$, (5) implies that in a neighbourhood of the origin in $\mathbf{C}$

$$
(u \circ F)(\lambda)=\langle\mathscr{L} u(0) \xi, \xi\rangle|\lambda|^{2}+o\left(|\lambda|^{2}\right) .
$$

(6) is implied directly by (7). Also from (7), applied to $F(\lambda)=\lambda \xi$, we deduce that Taylor's expansion of $u$ about the origin in $\mathbf{C}^{n}$ has the following form:

$$
u(z)=\langle\mathscr{L} u(0) z, z\rangle+o\left(\|z\|^{2}\right) .
$$

The first conclusion of the lemma follows form (8).

It is interesting to notice that if $u \in \operatorname{PSH}\left(\mathbf{C}^{n}\right) \cap \mathscr{C}^{2}(\{0\})$ is such that

$$
u(\lambda z)=|\lambda|^{2} u(z)
$$

for all $\lambda \in \mathbf{C}$ and $z \in \mathbf{C}^{n}$, then $u(z)=\langle\mathscr{L} u(0) z, z\rangle$ for all $z \in \mathbf{C}^{n}$ and hence $u^{1 / 2}$ is a seminorm. To see this, it is enough to apply the Laplace operator (with respect to $\lambda$ ) to both sides of $(\dagger)$. (See also the remarks following Theorem 1 in the Introduction.)

The families $\mathscr{S}(M, p)$ and $\mathscr{P}(M, p)$ have been used in the definitions of $S_{M}$ and $A_{M}$ respectively (see $\left.[8,1,2]\right) . S_{M}$ is defined by the formula

$$
S_{M}(\xi)=\sup \left\{\langle\mathscr{L} u(p) \xi, \xi\rangle^{1 / 2}: u \in \mathscr{S}(M, p)\right\}, \quad \xi \in T_{p} M
$$

For more information about the pseudo-metric $S_{M}$ see [8, 9]. 
Assume $\xi \in T_{P} M$. Let $V \subset \mathbf{C}$ be a neighbourhood of the origin and let $F: V \rightarrow M$ be a holomorphic mapping such that $F(0)=p$ and $F^{\prime}(0)=\xi$. Then we put

$$
L_{u}[\xi]=\limsup _{\substack{\lambda \rightarrow 0 \\ \lambda \neq 0}} \frac{\exp (u \circ F)(\lambda)}{|\lambda|}
$$

for any $u \in \mathscr{P}(M, p)$. It can be proved that the above definition is independent of the choice of $F$ (see $[1,2])$. (If $\exp (2 u) \in \mathscr{C}^{2}(\{p\})$, it follows directly form (6).)

Following Azukawa [1, 2] we define

$$
A_{M}(\xi)=\sup _{u \in \mathscr{P}(M, p)}\left\{L_{u}[\xi]\right\}, \quad \xi \in T_{p} M .
$$

From the definition of the extremal function $u_{M}=u_{M}(\cdot, p)$ we conclude (as in [2]) that for $\xi \in T_{p} M$

$$
A_{M}(\xi)=L_{u_{M}}[\xi]
$$

\section{Semicontinuity of $A_{M}$}

In this section we shall prove that if $M$ is a Stein manifold then $A_{M}$ is upper semicontinuous.

Lemma 3. If $M$ is a Stein manifold then $u_{M}: M \times M \rightarrow[-\infty, 0)$ is upper semicontinuous.

Proof. Since $M$ is a Stein manifold, there exists a smooth plurisubharmonic function $\psi: M \rightarrow \mathbf{R}$ such that the set $M_{c} \stackrel{\text { def }}{=}\{z \in M: \psi(z)<c\}$ is relatively compact in $M$ for each $c \in \mathbf{R}$. It follows from the definition of the extremal function $u_{M}$ that the sequence $\left\{u_{M_{j}}\right\}_{j \in \mathbf{N}}$ is decreasing and $\lim _{j \rightarrow \infty} u_{M_{j}}=u_{M}$ (see also [2] and [4]). Furthermore, as each of the sets $M_{j}$ is hyperconvex (in the sense of [4]), the functions $u_{M_{j}}: M_{j} \times M_{j} \rightarrow[-\infty, 0)$ are continuous by Theorem 4.14 in [4]. This means that the limit function $u_{M}$ is upper semicontinuous.

Lemma 4. If $M$ is a complex manifold such that $u_{M}: M \times M \rightarrow[-\infty, 0)$ is upper semicontinuous then $A_{M}: T M \rightarrow[0, \infty)$ is also upper semicontinuous.

Proof. Take $\xi_{0} \in T M$. Let $\pi: T M \rightarrow M$ be the canonical projection (i.e. $\left.\pi(\xi)=q \Leftrightarrow \xi \in T_{q} M\right)$. Let $\varphi: U \rightarrow \varphi(U) \subset \mathbf{C}^{n}$ be a holomorphic chart in a neighbourhood $U$ of $\pi\left(\xi_{0}\right)=p$. The chart $\varphi$ generates a chart $\tilde{\varphi}$ on $T M$ in the following way:

$$
\begin{gathered}
\tilde{\varphi}: \pi^{-1}(U) \rightarrow \varphi(U) \times \mathbf{C}^{n} \\
\tilde{\varphi}(\xi)=\left((\varphi \circ \pi)(\xi), a_{1}, \ldots, a_{n}\right) \Leftrightarrow \xi=\left.\sum_{j=1}^{n} a_{j} \frac{\partial}{\partial \varphi_{j}}\right|_{\pi(\xi)} .
\end{gathered}
$$


Define $g: \mathbf{C} \times \mathbf{C}^{n} \times \mathbf{C}^{n} \rightarrow \mathbf{C}^{n}$ by the formula $g(\lambda, z, a)=\lambda a+z$ and set $F(\lambda, \xi)=\varphi^{-1}(g(\lambda, \tilde{\varphi}(\xi)))$. Take a neighbourhood $V_{0}$ of $\xi_{0}$ and $r_{0}>0$ such that the mapping $\lambda \rightarrow F(\lambda, \xi)$ is well defined if $|\lambda|<r_{0}$ and $\xi$ is fixed in $V_{0}$. Let $F_{\xi}$ denote this mapping. Clearly $F_{\xi}(0)=\pi(\xi)$. In particular $F_{\xi_{0}}(0)=p$. Notice that if

$$
\xi=\left.\sum_{j=1}^{n} a_{j} \frac{\partial}{\partial \varphi_{j}}\right|_{\pi(\xi)}
$$

then $\left(d_{\pi(\xi)} \varphi\right)(\xi)=\left(a_{1}, \ldots, a_{n}\right)$. Therefore

$$
d_{0} F_{\xi}=\left(d_{\pi(\xi)} \varphi\right)^{-1} \circ d_{0}(\lambda \rightarrow g(\lambda, \tilde{\varphi}(\xi)))
$$

and hence

$$
F_{\xi}^{\prime}(0)=d_{0} F_{\xi}\left(\left.\frac{d}{d \lambda}\right|_{0}\right)=\left(d_{\pi(\xi) \varphi}\right)^{-1}\left(a_{1}, \ldots, a_{n}\right)=\xi
$$

Thus

$$
A_{M}(\xi)=\limsup _{\substack{\lambda \rightarrow 0 \\ \lambda \in \mathbf{C}}} \frac{\exp u_{M}\left(F_{\xi}(\lambda), \pi(\xi)\right)}{|\lambda|}, \quad \xi \in \pi^{-1}(U) .
$$

Suppose $A_{M}\left(\xi_{0}\right)<c$. By $(12)$, there is a number $r \in\left(0, r_{0}\right)$ such that

$$
\sup _{|\lambda|=r}\left(u_{M}\left(F_{\xi_{0}}(\lambda), \pi\left(\xi_{0}\right)\right)-\log |\lambda|\right)<\log c .
$$

This means that

$$
\sup _{|\lambda|=r} u_{M}\left(F_{\xi_{0}}(\lambda), \pi\left(\xi_{0}\right)\right)<\log r c .
$$

Because of upper semicontinuity of $u_{M}$ and continuity of $F$ and $\pi$ one can find a neighbourhood $V$ of $\xi_{0}$ such that $V \subset V_{0}$ and

$$
\sup _{|\lambda|=r} u_{M}\left(F_{\xi}(\lambda), \pi(\xi)\right)<\log r c, \quad \xi \in V .
$$

Therefore

$$
\sup _{|\lambda|=r}\left(u_{M}\left(F_{\xi}(\lambda), \pi(\xi)\right)-\log |\lambda|\right)<\log c .
$$

If $\xi$ fixed in $V$, the function $\left(\lambda \rightarrow u_{M}\left(F_{\xi}(\lambda), \pi(\xi)\right)-\log |\lambda|\right)^{*}$ is subharmonic in the disc $\left\{\lambda:|\lambda|<r_{0}\right\}$. Thus by the maximum principle for subharmonic functions

$$
\limsup _{\substack{\lambda \rightarrow 0 \\ \lambda \neq 0}}\left(u_{M}\left(F_{\xi}(\lambda), \pi(\xi)\right)-\log |\lambda|\right)<\log c
$$

for every $\xi \in V$. Consequently $A_{M}<c$ in $V$.

\section{Proof of THE THEOREMS}

The first statement of Theorem 1 follows directly from the definitions (9), (11) of the pseudo-metrics and from Lemma 2. Lemma 3 and Lemma 4 yield the second conclusion of the theorem. 
The estimates (3), (4) in Theorem 2 follow from the definition of $u_{\Omega}$ and from Lemma 2.

Now assume that $u_{\Omega} \in \mathscr{F}(\Omega, p)$ and the equality holds in (4) for all $\xi \in$ $T_{p} M$. Without loss of generality we may assume that $M$ is submanifold of $\mathbf{C}^{m}$ for some $m$. Since $\Omega$ is relatively compact in $M$, it is contained in an open ball with centre at $p$ and a positive radius $r$. It is clear that $\log \|z-p\|-\log r \leq u_{\Omega}(z)$ for all $z \in \Omega$. Let $N$ be a connected onedimensional complex submanifold of $\Omega$ such that $p \in N$ and $u \mid(N \backslash\{p\})$ is harmonic. Let $F: V \rightarrow M$ be a holomorphic parametrization of $N$ in a neighbourhood of $p$, such that $0 \in V \subset \mathbf{C}$ and $F(0)=p$. By applying (6) and the lower estimate for $u_{\Omega}$, we get

$$
\left\langle\mathscr{L}\left(\exp 2 u_{\Omega}\right)(p) F^{\prime}(0), F^{\prime}(0)\right\rangle \geq \lim _{\lambda \rightarrow 0} \frac{\|F(\lambda)-F(0)\|^{2}}{|r \lambda|^{2}}=\frac{\left\|F^{\prime}(0)\right\|^{2}}{r^{2}}>0 .
$$

Hence, (6) implies that

$$
1=\frac{\left\langle\mathscr{L} v(p) F^{\prime}(0), F^{\prime}(0)\right\rangle}{\left\langle\mathscr{L}\left(\exp 2 u_{\Omega}\right)(p) F^{\prime}(0), F^{\prime}(0)\right\rangle}=\lim _{\lambda \rightarrow 0} \frac{(v \circ F)(\lambda)}{\exp \left(2 u_{\Omega} \circ F\right)(\lambda)} .
$$

Therefore the function $\left(\left(v / \exp 2 u_{\Omega}\right) \mid N\right)^{*}$ is subharmonic on $N$ and attains its maximal value at $p$. Thus the maximum principle implies that $v=\exp \left(2 u_{\Omega}\right)$ on $N$. As $u_{\Omega} \in \mathscr{F}(\Omega, p)$, the same equality holds in $\Omega$.

If $n=1$ and the equality holds in (3) for one point $z \in \Omega \backslash\{p\}$, then the subharmonic function $v /\left(\exp 2 u_{\Omega}\right)$ has its maximum at $z$. Therefore-by the maximum principle-it is constant.

\section{REFERENCES}

1. K. Azukawa, Two intrinsic pseudo-metrics with pseudoconvex indicatrices and star-like circular domains, J. Math. Soc. Japan 38 (1986), 627-647.

2. The invariant pseudo-metric related to negative plurisubharmonic functions, Kodai Math. J. 10 (1987), 83-92.

3. E. Bedford and M. Kalka, Foliations and complex Monge-Ampère equations, Comm. Pure Appl. Math. 30 (1977), 543-571.

4. J. -P. Demailly, Mesures de Monge-Ampère et mesures pluriharmoniques, Math. Z. 194 (1987), 519-564.

5. M. Klimek, Complex foliations generated by (1, 1)-forms, Proc. Amer. Math. Soc. 91 (1984), 601-606.

6. __ Extremal plurisubharmonic functions and invariant pseudodistances, Bull. Soc. Math. France 113 (1985), 231-240.

7. L. Lempert, La métrique de Kobayashi et la représentation des domaines sur la boule, Bull. Soc. Math. France 109 (1981), 427-474.

8. N. Sibony, A class of hyperbolic manifolds, Ann. of Math. Stud., no. 100, Princeton Univ. Press, Princeton, N. J., 1981, pp. 357-372.

9. M. Suzuki, The intrinsic metrics on the domains in $C^{n}$, Math. Rep. Toyama Univ. 6 (1983), 143-177.

Department of Mathematics, University College Dublin, Belfield, Dublin 4, IRELAND 